\title{
Residual stresses of a magnesium alloy (AZ31) welded by the friction stir welding processes
}

\author{
A. Kouadri-Henni ${ }^{1 a}$, L. Barrallier ${ }^{2}$, Riad Badji ${ }^{3}$ \\ ${ }^{1}$ Université Européenne de Bretagne, France, INSA, LGCGM, EA 3913, F-35708 Rennes, FRANCE \\ ${ }^{2}$ Arts et Métiers Paris Tech, Mécasurf, JE 2504, F-13617 Aix-en-Provence, FRANCE \\ ${ }^{3}$ Research Center in Industrial Technologies CRTI, P.O Box 64, Cheraga, Algeria
}

\begin{abstract}
The objective of this study was to evaluate the residual stresses of FSW welding magnesium alloys (AZ31). The results show that the FSW processes lead to the formation of several distinct zones with differing mechanical properties. The residual stresses evolution have been explained by the heterogeneous modifications of the microstructure particularly a marked decrease in the grain size, a high modification of the crystallographic texture and the different anisotropic properties resulting from plasticity induced by the FSW process.
\end{abstract}

\section{Introduction}

Currently, industrial welding processes are rapidly evolving, this being driven by the need for process optimisation, cost effectiveness and ecological impact. It is for these reasons that friction stir welding (FSW) has started to appear in production workshops, notably in the automobile, aeronautical and mechanical industries. Furthermore these processes are being studied for welding materials that are reputed to be difficult to weld, such as aluminium or magnesium alloys. Magnesium alloys are potentially useful because of their low density, which is $35 \%$ less than that of aluminium, and their good mechanical characteristics [1,2]. Experimental work carried out in this area has shown that welding magnesium by FSW results in a better quality weld than that obtained by conventional methods (arc welding); however both methods require a precise evaluation of the parameters involved in the processes [3,4]. These studies have enabled a better understanding of the relationship between the welding processes, the weld quality, the microstructure and the residual stresses of the weld [3, 4]. Several studies have shown that the plasticity is a fundamental factor that governs the mechanical properties of the welded joints $[5,6]$. Most studies have shown the complexity of FSW and associated heterogeneities. From a mechanical point of view, considering the initial nature of the base metal (AZ31, AZ91, WE45, and AZ61), results has shown anisotropy in mechanical properties. These variation of mechanical properties have been linked principally to the initial fabrication process of the base metal and then to the welding process [6]. Welding processes equally produce residual stresses which are linked to the large deformation in FSW. For FSW peaks in residual tensile stress are observed in the TMAZ in the longitudinal direction and compressive residual stress peaks occur in a transversal direction in the fusion zone or nugget [6]. In the case of this study, a rolled magnesium alloy AZ31 was used for the welding tests to understand the evolution of microstructure and mechanical properties in particular the calculus of residual stresses.

\section{Principle of FSW}

The principle of FSW is based on the conversion of mechanical energy into thermal energy by friction generated by tool, which is generally threaded, on the material to be welded. This consists of a metal cylinder with a profiled and. threaded nib that is rotated at a high speed (Figure 1).

\footnotetext{
a Corresponding author: afia.kouadri-henni@insa-rennes.fr
} 


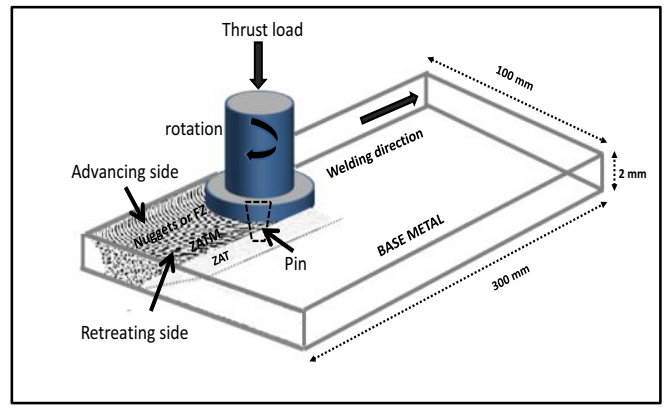

Figure 1.Principe of FWS process

The tool pin penetrates the plane of the joint at the interface between the two sheets to be welded and thoroughly mixes the metal of the two pieces in an operation which is a combination of forging and extrusion. The heat locally generated transforms the metal into a plastic state which allows the tool to be plunged into the material and then permits displacement along the line of the joint to form, after cooling, the weld. The melting point of the material is not reached in the process. The material undergoes a plasticizing process during the passage of the tool and a dynamic recrystallization during cooling $[7,8,9]$. At the end of this process one can distinguish several zones in the region of the weld in which the evolution of the microstructure is controlled by a complex interaction between plastic strain, recovery and recrystallization. These interactions will also include homogeneous and heterogeneous precipitation phenomena depending on the nature of the alloy.

\section{Experimental methods}

\subsection{Sample presentation}

The alloy used in the study FSW welding was a ternary magnesium - aluminium - zinc alloy with the designation AZ31 according ASTM standards. AZ31 magnesium alloy was chosen due to its good rolling capabilities. $2 \mathrm{~mm}$ thick plates were hot rolled by several passes and then levelled and $300^{\circ} \mathrm{C}$ annealed. Table 1 shows the chemical specification of the AZ31 magnesium alloy supplied.

Table 1: Principal chemical composition of AZ31 alloy in base metal, BM, (EDS analysis) [10]

\begin{tabular}{|c|c|c|c|c|c|}
\hline BM & Al & Zn & Mn & Si & Cu \\
\hline Element & $2.5-$ & $0.6-$ & $0.2-$ & $<0.05$ & $<0.008$ \\
(weight \%) & 3.5 & 3.4 & 0.6 & & \\
\hline
\end{tabular}

AZ31 magnesium alloys used were rolled to produce $2 \mathrm{~mm}$ thin plates from $100 \mathrm{~mm}$ ingots which were hot rolled with several passes using a 4 rollers Quarto rolling mill. Strain rates between 5$20 \%$ (thickness reduction per pass) and rolling speeds between $0.5-2 \mathrm{~m} / \mathrm{s}$ were used. A strong basal texture resulted from this process. These plates were used as raw material for welding and as samples for base material analysis. For the experimental study performed within the framework of our research, samples were machined into parallelepidic shapes with dimensions of $300 \times 100 \times 2 \mathrm{~mm}^{3}$.

\subsection{Parameters of the welding process}

Two plates were welded together side by side using a FSW process. To validate the optimisation strategy, a plan of experiments has been carried out following a simplified plan of Tagushi.

FSW processing was carried out with machines located at the Welding Institute (IW-G-ESAB). The processing parameters influencing the weld quality were studied. The visual aspect of the FSWelds was improved with increasing the load applied (F), welding speed $(\mathrm{V})$ and tool rotation rate $(\mathrm{W})$ with a smaller flash, less surface corrosion and less tunneling phenomena occurring. Experiments performed showed that increasing welding speed (V) at a constant tool rotation rate (W) resulted in internal voids and lack of bonding caused by the insufficient material flow. For a constant welding speed, low tool rotation rates lead to the formation of internal voids because the frictional heat was not sufficient to promote material flow. These defects disappeared with increasing $\mathrm{W}$, but with a further increase, internal voids, lack of bonding and surface cracking occurred due to excess expelled material. These results are consistent with the literature $[5,6$, $7,8]$. The tool used had a $5 \mathrm{~mm}$ pin diameter and $10 \mathrm{~mm}$ shoulder. The process parameters that were varied were the welding speed (V) and tool rotation rate $(\mathrm{W})$. Various clamping systems and welding pressures were tested. The optimized domains are summarised in table 2. After welding, the welded samples had dimensions of $300 \times 200 \times 2 \mathrm{~mm}^{3}$.

Table 2: Welding FSW parameters choice

\begin{tabular}{|c|c|c|}
\hline $\begin{array}{c}\text { Welding } \\
\text { pression } \\
(\mathbf{k N})\end{array}$ & $\begin{array}{c}\text { Welding speed V } \\
(\mathbf{m m} / \mathbf{m i n})\end{array}$ & $\begin{array}{c}\text { Tool rotation } \\
\text { rate W (RPM) }\end{array}$ \\
\hline 1.5 & 90 & 1200 \\
\hline
\end{tabular}

\subsection{Location of the analysed zones}


Figure 2 shows the location of the zones that were analysed in the work pieces welded by FSW.

Measurements were carried out in each of the zones modified by the welding process; the measurements were uniquely at the surface and ranged from the centre of the weld to the base metal. In our case, with the FSW process, two measures were carried out on either side of the fusion zone which consisted of two zones: the central stir friction zone (SWZ, zone 4); the thermo mechanically affected zone (TMAZ) on the left of the weld, advancing side, (TMAZ, zone 3); and the TMAZ to the right, the retreating side, referred to as TMAZ, zone 5. The HAZ to the left and right of the weld are respectively referred to as HAZ, zones 2 and 6 and finally the base metal BM, zones 1 and 7 .

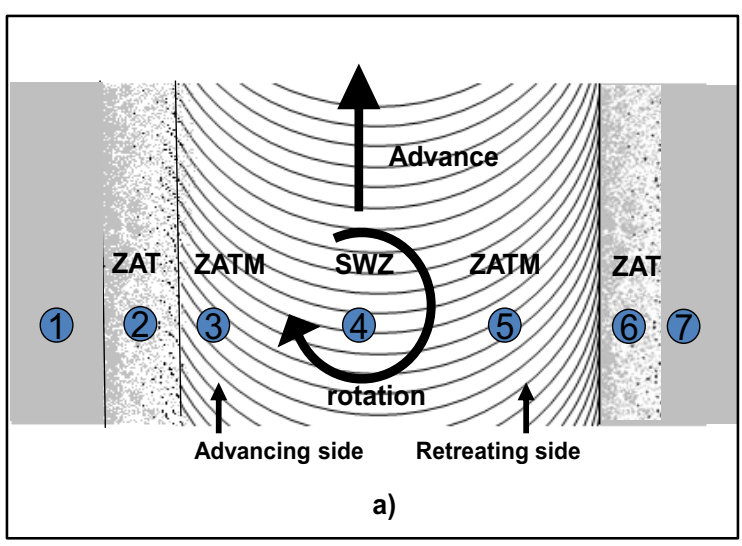

Figure 2: Location of the analysed zones

\subsection{Metallurgical analysis}

Before welding, each sample was machined to remove the oxide layer and cleaned with acetone. After mechanical polishing to a mirror finish, the welds were examined by optical microscope. The microstructure of the magnesium alloy was exposed by Nital Etchant (4\% and 20\% HNO3, $96 \%$ $\left.\mathrm{C}_{2} \mathrm{H}_{5} \mathrm{OH}\right)$ and by the reaction of Keller Etchant (2.5 $\mathrm{ml}$ of HNO3, $1.5 \mathrm{ml}$ of $\mathrm{HCl}, 1.0 \mathrm{ml}$ of $\mathrm{HF}$ and 95 $\mathrm{ml}$ of water). Observations were also made using a scanning electron microscope (SEM); point chemical analysis was carried out on the samples using an electron microprobe (EDS).

\subsection{Mechanical analysis}

\subsubsection{Hardness measurement}

The micro hardness was measured by a Vickers indenter under a load of $100 \mathrm{~g}$ on cross sections applied for $15 \mathrm{sec}$. A program on a conventional optical microscopy measured the diagonal lines of the mark. A series of measurements were carried out close to the surface, at every location, from the SWZ, FZ and to the BM.

\subsubsection{Tensile tests}

The evaluation of the mechanical behaviour of the weld was carried out using transverse tension test. Dumbbell specimen preparation was performed using water jet cutting. The welded plates were cut transversely across the weld. The lateral section was then polished to avoid crack initiation during tensile test. An Instron machine with mechanical grips and a $50 \mathrm{kN}$ load cell was used and tensile tests were performed at $5 \mathrm{~mm} / \mathrm{min}$ strain rate.

\subsubsection{Calculation of residual stresses}

The method used to calculate the residual stresses by X-ray diffraction uses the distance between crystallographic planes as an internal strain gauge. The diffractometer used for these studies was equipped with a 4 circle goniometer - (Seifert MZ VI - TS) and a PSD detector. The X-ray source was a $1 \mathrm{~mm}$ collimator and the wave length used was $\mathrm{Cr}-\mathrm{K} \alpha$. The intensity and voltage of the source were $30 \mathrm{~mA}$ and $40 \mathrm{kV}$ respectively. A masking system was used to limit the irradiated zone to $0.8 \mathrm{~mm}$. Under these conditions the depth of penetration into the AZ31 was estimated to be $7 \mu \mathrm{m}$. The calculation of residual stress required several steps:

\section{a) Identification of crystalline phases}

Identification of the phases was carried out by indexing diffraction peaks. The position of the peaks was defined by optimisation of parameters of the pseudo-Voigt function $\left(K \alpha_{1}\right.$ and $\left.K \alpha_{2}\right)$ of the diffraction intensities. The information thus obtained was used to determine which family of crystalline planes would be used as the internal gauge and consequently the settings to adopt for the measurement of strain $\varepsilon_{\phi \psi}$. In this study, only the $\alpha$ phase was taken into consideration.

b) Calculation of Orientation Distribution Function (ODF)

The AZ31 base metal was in hot rolled condition. Using this deformation process, highly textured resulting material was expected. FSW induced high deformation gradients which may cause changes in crystalline orientations. The study of the texture evolution is then required to understand the anisotropic characteristic of the welds and its influence on mechanical properties. The texture of the base metal and the texture evolution in FSWelds was studied using XRD techniques. Analysis of the structure was only carried out for the predominant phase: the $\alpha-\mathrm{Mg}$ matrix. The textures were measured by the choice of 5 pole figures corresponding to the diffraction planes of the 
principal $\alpha-M g$ phase: the prismatic plane $\{10 \overline{1} 0\}$, basal $\{0002\}$, pyramidal $\{10 \overline{1} 1\}$, planes of the type $\{10 \overline{1} 2\}$ and $\{11 \overline{2} 0\}$. The diffraction intensities, expressed as the number of counts per second, were measured by the variation of two angles: the polar angle $\psi$, varying from 0 to $70^{\circ}$ starting in the axial direction of the sample and a step size of $5^{\circ}$; the azimuth angle $\phi$, varying from 0 to $360^{\circ}$ with a step size of $10^{\circ}$. The ODF was then calculation from the 5 pole figures $[10,11$, 12]. We extracted the information's such as the volume fraction of the principal orientation from the ODF (example is given in figure 3 for the zones welded FSW process).

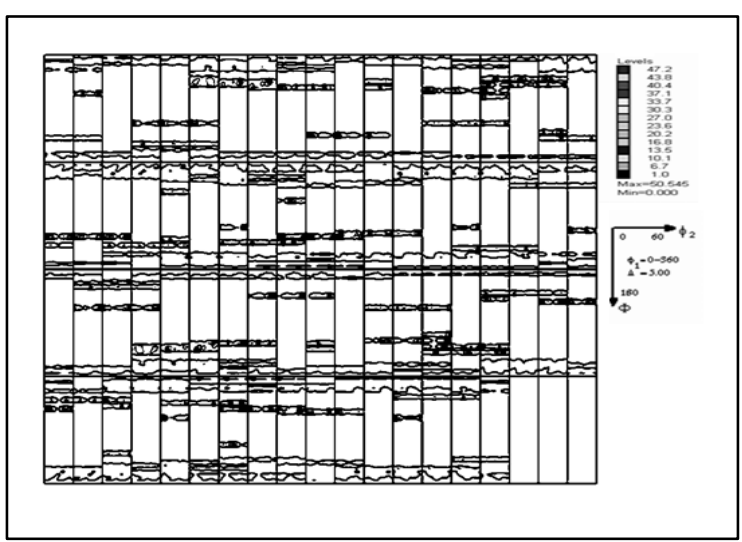

Figure 3: ODF of the welded zone by FSW process

Every orientation and fraction volume in every zone has been extracted from the ODF. We introduced the data in the calculation of residual stresses in the case of a presence of a texture.

\section{c) Strain Measurement $\varepsilon_{\phi \psi}$}

The strains $\varepsilon_{\phi \psi}$ were deduced from the angular shift of the diffraction peaks. For the measurement of strain the diffraction plane chosen was the plane $\{10 \overline{1} 3\}$ of the magnesium phase. The measurements were carried out by the simultaneous variation of 11 polar angles $\Psi$ varying from $\pm 65^{\circ}$ with a $\sin ^{2} \Psi$ distribution, and 6 azimuth angles varying from 0 to $360^{\circ}$.

\section{d) Calculation of residual stresses}

When a material is not textured, therefore isotropic, the residual stresses were calculated by the $\sin ^{2} \Psi$ law [10].

$$
\varepsilon_{\phi \psi}=\frac{1}{2} S_{2} \sigma_{\phi} \sin ^{2} \psi+S_{1}\left(\sigma_{11}^{\mathrm{S}}+\sigma_{22}^{\mathrm{S}}\right)
$$

When the material is textured, the residual stresses were calculated taking into account the grain orientation: the orientation distribution function (ODF).

$$
\varepsilon_{\phi \psi}(\mathrm{hkl})=\mathrm{F}_{\phi \psi}^{\mathrm{hkl}}(\phi, \Psi, \Omega, \mathrm{s}(\Omega), \mathrm{S}, \mathrm{ODF}): \sigma_{\mathrm{ij}}
$$

The components of the compliance tensor $S_{1}$ and $S_{2}$ were calculated with the aid of the elastic constants of a mono-crystal of magnesium taken from published literature (equation 1). The values used in the calculation of residual stress were as follows: $\mathrm{S}_{1}\left(-5,5810^{-6} \mathrm{MPa}^{-1}\right)$ and $\mathrm{S}_{2}(26.04$. 10 $\left.{ }^{6} \mathrm{MPa}^{-1}\right)[13,14,15]$. In the case of textured zones, the residual stresses were calculated taking into account the anisotropic polycrystalline constants. In this case the radio-crystallographic elastic constants $\mathrm{F}_{\phi \psi}^{\mathrm{hk} l}$ (equation 2) were determined from the ODF. In both cases a homogeneous KrönerEshelby self-consistent model was used [14, 15]. With the X-ray measurement technique used only the superficial layer of the metal (several microns) interact with the X-ray beam. The stress state was taken as plane stress $\left(\sigma_{3}=0\right)$.

\section{Results and discussion}

The FSW welding processes result in different microstructures and mechanical properties.

\subsection{General properties of the base metal}

The AZ31 base metal revealed a fine equiaxed grain structure, with very few deformation twins. Two grain size populations could be observed $(5 \mu \mathrm{m}$ and $15 \mu \mathrm{m}$ ) and the average grain size is $10 \mu \mathrm{m}$ (figure 4).

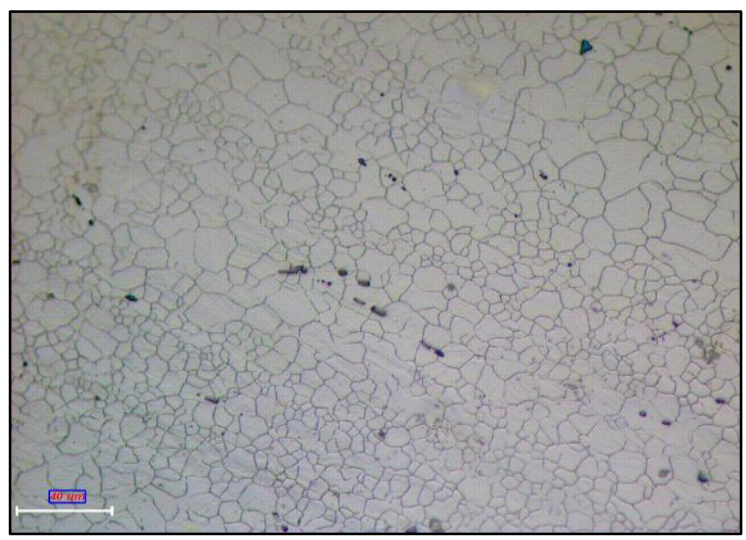

Figure 4: Microstructure of base metal

AZ31 optical micrograph study showed that the microstructure consisted in principally of a primary $\alpha-\mathrm{Mg}$ phase structure. 


\subsection{Evolution of the microstructure in the welded zones}

In the case of the FSW process, the base metal which is remote from the weld site is not subjected to any significant deformation or heating; that is to say it conserves its original properties. Figure 5 shows the different zones resulting from the FSW process.

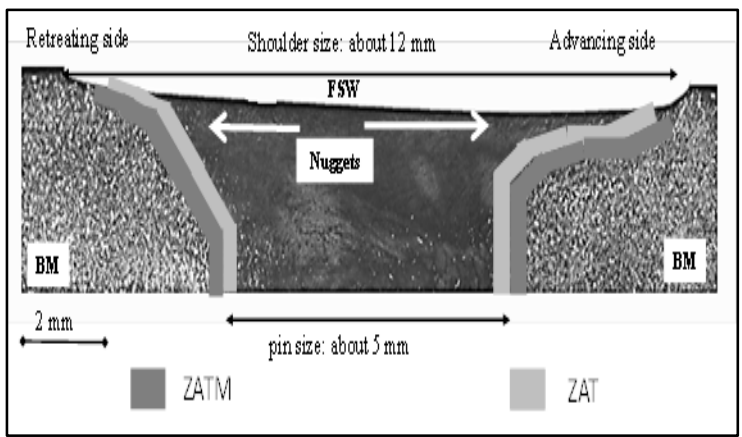

Figure 5: Microstructure of FSWeld section

The figure 6 shows the microstructure evolution:

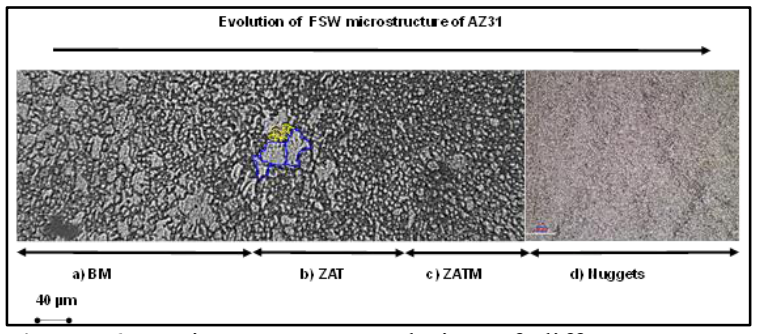

Figure 6: Microstructure evolution of different zones resulting from the FSW process.

The FSW process leads to a welded zone with two distinct zones: The HAZ and the friction zone. The latter zone is comprised of two zones: the thermomechanically altered zone TMAZ (figure 6c) and the stir welded zone, SWZ (6d), equally referred to as the nugget. In the FSW process, the HAZ (6b) is subjected to heating without notable deformation.

A large microstructure gradient is obtained by FSW process. Dynamic recrystallization occurs in the FSW nugget, leading to a fine grain size structure (about $2 \mu \mathrm{m}$ ). In the TMAZ, microstructure consists in not fully recrystallized grains. The TMAZ grain size ranges between 2 to $15 \mu \mathrm{m}$. The zone, situated at the boundary between the base metal and the highly deformed zone (TMAZ) can be characterised by the similarity of the grains to those of the base metal and the fine grains, interposed between the coarse grains, resulting in a dynamic recrystallization.

\subsection{Results Orientations Overlap Volume Fraction}

Superficial texture analysis was performed using laboratory XRD. Measurements were made in the nugget, TMAZ, HAZ and BM. The pole figures were examined with respect to the surface and central regions on both sides of the centerline (i.e., on the advancing and retreating sides) $\mathrm{BM}$ and HAZ. The volume fraction was extracted from ODF.

\subsubsection{BM and HAZ}

The base metal pole figures showed a strong texture, with the $\{0002\}$ basal plane normal, parallel to the normal direction (ND), which is the typical hot rolling texture of magnesium alloys [4]. Concerning the HAZ, the results show a texture near of that of the BM. The fraction volume is slowly low compared of BM. These results (table 3) are reasonable because the HAZ retains the same grain structure as the parent material. Indeed, this zone does not undergo any plastic deformation but experiences thermal cycling. This temperature leads to some microstructural change but is not sufficient to modify the crystallographic texture of the materials. ODF calculation indicates that more than about 90 pct of the grains are oriented.

Table 3: Orientations volume fraction in the $\mathrm{BM}$ and HAZ obtained by FSW process

\begin{tabular}{|c|c|c|c|c|}
\hline Volume & Delta & Delta & Delta & \\
\hline Fraction & Phi1 & Phi & Phi2 & Orientation \\
\hline 89.35 & 205.00 & 55.00 & 12.50 & 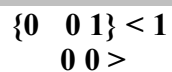 \\
\hline 1.35 & 10.00 & 10.00 & 10.00 & $\begin{array}{ccc}\left\{\begin{array}{ccc}0 & 0 & 1\end{array}\right\}<1 & -2 & 0\end{array}$ \\
\hline 9.33 & \multicolumn{4}{|c|}{ The Rest Volume Fraction } \\
\hline 90.70 & \multicolumn{4}{|c|}{ Orientations Overlap Volume Fraction } \\
\hline
\end{tabular}

\subsubsection{Welded Zone}

With FSW process, the ODF calculation indicates a preferential orientation of the grains in the nuggets (table 4a) with the $\{002\}<210>$ orientation (about $90 \%$ ). which correspond to the three Euler angles $\left(\varphi_{1}=205^{\circ}, \phi=55^{\circ}, \varphi_{2}=12.5^{\circ}\right)$.

Tables 4a: Orientations volume fraction in the nuggets obtained by FSW process

\begin{tabular}{|c|c|c|c|c|}
\hline Volume & Delta & Delta & Delta & \\
\hline Fraction & Phil & Phi & Phi2 & Orientation \\
\hline 94 & 205.00 & 55.00 & 12.50 & $\begin{array}{lll}\left\{\begin{array}{lll}0 & 0 & 2\end{array}\right\}< \\
2 & 1 & 0\end{array}$ \\
\hline 4.63 & 45.00 & 45.00 & 45.00 & $\begin{array}{l}\left\{\begin{array}{lll}0 & 0 & 1\end{array}\right\}<7 \\
1-1>\end{array}$ \\
\hline 2.37 & \multicolumn{4}{|c|}{ The Rest Volume Fraction } \\
\hline 97.63 & \multicolumn{4}{|c|}{ Orientations Overlap Volume Fraction } \\
\hline
\end{tabular}


In the nugget zone, the orientation with the $\{002\}$ basal plane normal parallel to the normal direction (ND) is the typical friction texture of magnesium alloys. By comparing the ODF calculation (Tables $4 \mathrm{~b}$ and $4 \mathrm{c}$ ) along both sides of the central region, i.e., in the fracture region (TMAZ), the results show that most of the TMAZ has orientation components with a strong tendency for the (001) basal plane to align with the surface of the hard pin of the welding tool. This zone presents only the principal orientation of the (0002) basal plane. These texture components were rotated around the 'normal direction', the direction of the axis of the pin. The tilt is different along the side. In the literature, these local orientation evolutions are explained as being mainly due to shear plastic flow along the pin column surface [6,7]. These zones were deformed and influenced by heat and high plastic deformation. Our results show that these zones are characterized by the modification of a crystallographic texture. The pole figures show that a texture developed along the top surfaces of the welded zones, with (0002) parallel to the plate surface.

Tables 4b: Orientations volume fraction in TMAZ (advancing side) zone obtained by FSW process

\begin{tabular}{|c|c|c|c|c|}
\hline Volume & Delta & Delta & Delta & \\
\hline Fraction & Phil & Phi & Phi2 & Orientation \\
\hline 11.14 & 45.00 & 42.50 & 40.00 & $\begin{array}{ccc}\left\{\begin{array}{rrr}0 & 0 & 1\end{array}\right\}< \\
14 & 15 & 0>\end{array}$ \\
\hline 83.97 & 45.00 & 45.00 & 40.00 & $\begin{array}{c}\left\{\begin{array}{lll}0 & 0 & 1\end{array}\right\}< \\
1\end{array}$ \\
\hline 4.89 & \multicolumn{4}{|c|}{ The Rest Volume Fraction } \\
\hline 95.11 & \multicolumn{4}{|c|}{ Orientations Overlap Volume Fraction } \\
\hline
\end{tabular}

Tables 4c: Orientations volume fraction in TMAZ (retreating side) zone obtained by FSW process

\begin{tabular}{|c|c|c|c|c|}
\hline Volume & Delta & Delta & Delta & \\
\hline Fraction & Phil & Phi & Phi2 & Orientation \\
\hline 76.60 & 45.00 & 42.50 & 40.00 & 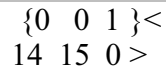 \\
\hline 19.37 & 45.00 & 45.00 & 40.00 & 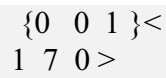 \\
\hline 4.03 & \multicolumn{4}{|c|}{ The Rest Volume Fraction } \\
\hline 95.97 & \multicolumn{4}{|c|}{ Orientations Overlap Volume Fraction } \\
\hline
\end{tabular}

The results show that most of these zones had a texture with a strong tendency for the (0002) basal plane to align with the surface of the hard pin of the welding tool. The appearance of other texture components in the stirred zone in addition to that of the basal plane indicates that friction stir welding leads to several mechanisms in the stirred zone: a shear deformation process and dynamic recrystallization.

\subsection{Evolution of micro-hardness}

Average values of micro hardness measures in the different zones are shown in figure 7.

In the case of the FSW process the average micro hardness in the fusion zone is $85 \pm 2 \mathrm{HV}$ which is slightly less than that of the base metal: 90 $\pm 5 \mathrm{HV}$. The micro hardness decreases slightly in the fusion zone and in the TMAZ $(82 \pm 2 \mathrm{HV})$; the slight decrease in the HAZ, where the grain sizes are identical to those in the BM, can be explained by the fact the material has been submitted to a thermal annealing cycle $[6,8]$.

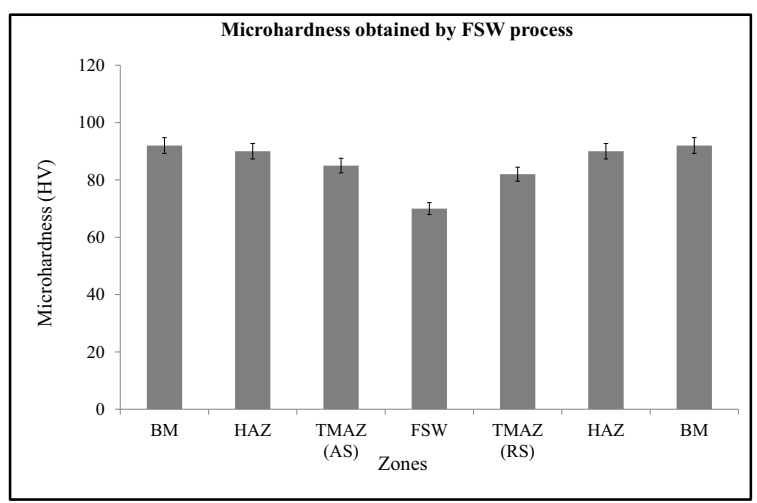

Figure 7: Micro hardness measured in the difference zones

In the welded zone there is a slight reduction in micro hardness despite the fact that the grain size is greatly reduced; the values obtained do not obey the Hall and Petch Law [10]. Within the welded zone the variation of micro hardness can be explained by the fact that the high degree plastic deformation and heating are conducive to the phenomenon of dynamic recrystallization resulting from dynamic recovery. This effect predominates over the reduction in grain size and leads to a reduction in the density of dislocation resulting from the restoration phenomenon. This phenomenon occurs in the case of non-age hardened alloys that have been heavily strain hardened as in the case of AZ31. Close the TMAZ / nugget the hardening due to work hardening resulting from the action of heavy deformation that gives rise to a partial recrystallization and splitting up of the intermetallic which could explain why the micro hardness remains relatively constant compared with the large decrease in the grain size.

\subsection{Evolution of residual stresses}

Figure 8 shows the average calculated values of the longitudinal and transversal residual stresses in the various zones welded by FSW. 


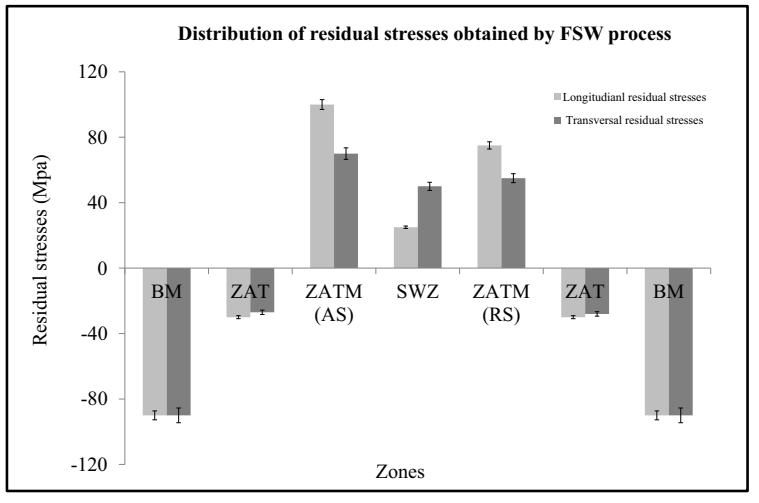

Figure 8: Evolution of residual stresses

The base metal has a compressive residual stress of the order of $100 \mathrm{MPa}$. In the zones welded the FSW process highly modifies the distribution of residual stress in the various zones. The HAZ has weak compressive residual stresses and is of similar magnitude in the longitudinal and transversal directions to the weld seam (30 MPa). In both the TMAZ and FZ the residual stresses are tensile. The residual stress profile in both of these zones shows two pronounced peaks in the TMAZ whereas the centre of the FSW has very low tensile residual stresses. Furthermore the profile is partially nonsymmetrical transversal to the weld. It appears that the TMAZ does not have the same behaviour on the advancing side (AS) and the retreating side (RS) of the weld. The advancing side of the weld has a higher level of residual stress in comparison with the retreating side. This variation in stress corresponds with the asymmetrical plastic deformation imposed on the material which is inherent in this process: the force causing plastic deformation is higher on the AS side of the weld $[11,26]$. The heterogeneity of stresses is in turn linked to the FSW method which induces local and different plastic deformations in the weld zone. These heterogeneities can equally be attributed to the formation of a crystallographic texture with differing orientations depending on the zone analysed [12] and to the presence of shear bands.

\section{Conclusion}

The objective of this study was to compare the local properties: microstructure, micro hardness, and residual stresses) of the same metal: thin sheets of magnesium alloy AZ31.

The FSW process does not lead to chemical modifications and significant phase changes. On the other hand it does lead to the phenomenon of partial recrystallization in the TMAZ and total recrystallization in the SWZ. Furthermore the welded zone could be characterised by tensile residual stresses and a heterogeneity depending on which zone was analysed. These variations could be attributed to the presence of a textured crystallography and shear bands which cause local plastic strain within these zones. The dissymmetry of stresses between the advancing and retreating sides of the joint can be attributed to the higher plastic force on one side of the weld and is inherent in the FSW process.

\section{References}

1. C.E.D. Rowe and W.M. Thomas, Advances in tooling materials for friction stir welding, Technical report, TWI Ltd Cambridge, UK.

2. R.S. Mishra, Z.Y. Ma, Mater. Sc. and Eng. R 50, 1-78 (2005)

3. H. Zhang, S. B. Lin, L. Wu and J. C. Feng. Act. metta .sin.17, 747-753(2004)

4. M.M. Avedesian and H. Baker, ASM Specialty Handbook, (1999)

5. R.S. Mishraa and Z.Y. Ma. Mater. Sc. and Eng. Reports 50, 1-78(2005).

6. T. Nagasawa, M. Otsuka, T. Yokota, and T. Ueki. Chapter Magnesium Technology 2000. The Miner., Metal., Mater. Society, 383-387 (2000).

7. A. Scialpi, L.A.C. De Filippis, P. Cavaliere, Mater. and Des.28, 1124-1129(2007).

8. K. Elangovan, V. Balasubramanian, Mater.ials Sc. and Eng. 459A, 7-18(2007).

9. Mustafa Boz, Adem Kurt, Mater. and Des. 25, 343-347(2004).

10. A. Kouadri, L. Barrallier, Mater. Sci. Eng. 429A, 11- 17(2006).

11. W. Woo, H. Choo, D.W. Brown, P.K. Liaw, Z. Feng, Scr. Mater. 54, 1859-1864(2006).

12. A.P. Reynolds, Elizabeth Hood, Wei Tang, 21S, Scr. Mater.52, 491-494(2005)., p. 491-494.

13. F. Caleyo, T. Baudin, M.H Mathon and R. Penelle, Eur. Phys. J. AP, 15, 85-96(2001).

14. H.R. Wenk, K. Pawlik, J. Pospiech and J.S Kallend, Textures and microstructures 22, 233260(1994).

15. K. Pawlik, J. Pospiech, K. Lücke K., Proceeding of ICOTOM-8, Santa Fe, USA, 531(1987). 